Gdańsk 2019, Nr. 41

https://doi.org/10.26881/sgg.2019.41.03

Pierre-Yves Modicom

Universität Bordeaux-Montaigne

https://orcid.org/0000-0002-9457-2442

\title{
Deutsche Modalpartikeln in Befehlssätzen: Intersubjektivität, Deontik und Satzmodusspezifizierung
}

Der Beitrag bietet einen Überblick über den Gebrauch von Modalpartikeln (MPn) in deontischen Sprechakten an. Wie verhalten sich MPn, die oft dem epistemischen Bereich zugeordnet werden, in Äußerungen ohne epistemische Komponente? Am Beispiel von doch skizziere ich zunächst ein Modell zur Verortung der modalen und illokutiven Interpretationsbreite von MPn. Dann wird der Gebrauch von $J A$, eben, halt und rubig in Befehlsätzen untersucht. Schließlich werden die Sonderfälle nur und mal behandelt.

Schlüsselwörter: Deontik, Modalpartikel, Satzmodus, Illokution.

German Modal Particles in Directive Speech Act Clauses: Intersubjectivity, Deontic Modality und Sentence Mood Specification. - This contribution on German is concerned with the use of Modal Particles (MPs) in speech acts characterized by strict deontic modality, while MPs are often seen as representing an intersubjective dimension of epistemicity. First, taking the example of doch, I sketch a model of semantic and pragmatic variation disentangling the various dimensions of modal and illocutionary variation from the stable lexical meaning of MPs. Then I turn to MPs $J A$, eben, halt and rubig in clauses expressing directive speech acts. Finally, I discuss the problematic cases of nur and mal.

Keywords: Deontic speech acts, imperatives, Modal particles, sentence mood, illocution

\section{Einleitung ${ }^{1}$}

Die meisten Studien über Modalpartikeln (MPn) befassen sich mit ihrem Verhalten in epistemisch geprägten Sprechaktsorten: Assertionen und Interrogationen verschiedener Sorten (Entscheidungsfragen, W-Fragen, Vletzt-Fragen...). Das Ziel dieses Artikels ist, den Beitrag von MPn in deontischen Äußerungen zu untersuchen. Solche Fälle stellen eine Herausforderung für jene Beschreibungsansätze dar, die MPn als Exponenten einer Abschattung epistemischer Modalität bezeichnen. Die wichtigsten MPn in Befehlsätzen sind doch, JA, eben, halt, rubig, und (sofern sie zu den MPn gezählt werden) nur und mal. Der Beitrag liefert einen Überblick über ihre

1 An dieser Stelle möchte ich mich bei allen Teilnehmern der Tagung in Gdańsk herzlich bedanken, sowie bei den Herausgebern und einem anonymen Gutachter. 
Gebrauchsbedingungen und ihre kontextuelle illokutive Varianz. Ausgehend von einer bedeutungsminimalistischen Perspektive wird ein Modell skizziert, in welchem die Art der Modalität (Epistemik vs. Deontik) erst durch die Kombination mit dem Satzmodus bestimmt wird. Die Modellierung der Bedeutung erfolgt nicht auf der Ebene der Sprechaktteilnehmer, sondern auf derjenigen der modalen Bewertungsinstanzen. Erst in einem zweiten Schritt erfolgt die illokutive Interpretation der Kombination aus Satzmodus und Partikel. Dieser Beschreibungsansatz wird zunächst am Beispiel von doch vorgestellt (Abschnitt 1). Anschließend wird der semantische Beitrag von JA, eben, halt und rubig entlang dieser Linien vorgestellt (Abschnitt 2). Die Grenzen dieses Modells werden abschließend am Beispiel von nur und mal besprochen, wobei die Frage aufgeworfen wird, ob dies nicht ein zusätzliches Argument gegen ihre Zuordnung zu den MPn bildet (Abschnitt 3).

\section{Modalpartikeln und Sprechaktsensitivität}

\subsection{Zum Status der Sprechaktteilnehmer in der semantischen Beschreibung von Modalpartikeln}

In der Literatur über MPn herrscht größtenteils Konsens über die Annahme, dass MPn die Erwartungen eines Sprechaktteilnehmers bezüglich der intersubjektiven Ratifizierung des Gehalts der Aussage markieren. Spätestens seit DoHERTY (1985) wird dieser intersubjektive Abgleich sehr oft auf Epistemik zurückgeführt: Was ich oben „Ratifizierung des Gehalts der Aussage" genannt habe, sei eigentlich eine Antizipation auf das Bekenntnis des Hörers zur Wahrheit des propositionalen Gehalts $\mathbf{p}$.

Dass es immer um eine Einfühlung des Sprechers in die propositionalen (bzw. epistemischen) Einstellungen des Hörers geht, wurde allerdings mehrmals bestritten, unter anderem wenn das Verhalten von MPn in Fragesätzen in die Analyse einbezogen wurde. Die Analyse von wobl bekräftigt diesen Verdacht. Kwon (2005), ZimmermanN (2008), GutZmanN (2011,2015: 255) und andere haben jeweils eine Umkehrung der epistemischen Perspektive zwischen wohl-Assertionen und wohl-Fragen gemerkt. Der Unsicherheitseffekt, der mit dem Gebrauch von wobl einhergeht, bezieht sich in Assertionen auf den Sprecher:

(1) Digitale Kommunikation kann mitgelesen, mitgehört werden. Dieser Leitsatz, diese Warnung hat sich in den vergangenen Monaten wohl bei vielen im Hinterkopf festgesetzt. Zu deutlich waren die vielen Veröffentlichungen, die auf Edward Snowden zurückgehen, den früheren USGeheimdienstmitarbeiter. (DR 070913-03)²

2 Beispiele mit dem Hinweis DR stammen aus meinem eigenen Forschungskorpus (s. Modicom 2016). Dieses Korpus besteht aus den Transkriptionen von 20 Stunden Radiogesprächen. Es handelt sich dabei um sämtliche Interviews von Deutschmuttersprachlern, die im Morgenprogramm der öffentlich-rechtlichen Bundesradiosender Deutschlandfunk und DeutschlandRadioKultur zwischen dem 16. August und dem 23. September 2013 übertragen wurden, also im letzten Monat des Bundestagswahlkampfes 2013. Die Audiodateien wurden über einen Podcast zur Verfügung gestellt und DeutschlandRadio erteilte eine freundliche Genehmigung zur wissenschaftlichen Benutzung der Daten. Auch wenn Zögerungen, Fehler und Wiederholungen transkribiert wurden, war die Untersuchung, um die es damals ging, nicht gesprächslinguistisch orientiert, daher die Nicht-Beachtung der GAT- bzw. HIAT-Transkriptionsstandards. Das Korpus umfasst 208.550 Tokens und wurde mit der Software TXL untersucht. Für eine qualitative Besprechung, s. Modicom (2016: 95-109). 
In adressierten Fragen aber geht es vielmehr um den Sicherheitsgrad des Hörers. KwoN (2005: 165) schreibt:

„Während wohl in assertiven Fragesätzen immer die sprecherseitige Unsicherheit signalisiert, kann sich wohl in w-Fragesätzen sowohl auf die sprecherseitige Unsicherheit als auch auf die hörerseitige Unsicherheit beziehen."

Das sieht man auch am Beispiel von Vletzt-Fragen, die sehr oft eine MP wohl zulassen (dazu s. GutZmann 2011).

(2) Ob er wohl noch kubanische Zigarren mag? (Zit. TRUCKensiodt 2006: 274)

Dieser Wechsel einspricht dem epistemischen Rollenwechsel zwischen Sprecher und Hörer (Zimmermann 2008). Es sollte daher in Erwägung gezogen werden, ob MPn nicht so sehr eine Sprecher-Hörer-Beziehung markieren, als das Verhältnis zwischen dem epistemischen Richter, dem Wahrheitswert von p, und ggf. einer zweiten modalen Instanz, die ich im Folgenden den Ratifizierer nennen werde.

Nun ist wohl eine eigentümliche MP, denn sie befasst sich vielmehr mit der (epistemischen?) Bewertung von $\mathbf{p}$, als mit der Antizipation der Ratifizierung. In Modicom (2016) habe ich eine entsprechende Unterscheidung gemacht zwischen subjektiven MPn (wohl, denn) und intersubjektiven MPn (ja, doch, schon, eben...). Die Frage ist, ob sich der deskriptive Sprung von Sprechaktteilnehmern auf getrennte modale Instanzen auch für intersubjektive MPn generalisieren lässt. Da manche dieser Partikeln auch in nicht-epistemischen Satzmodi ${ }^{3}$ zulässig sind, sollte auch der Begriff des epistemischen Richters hier hinterfragt werden. Im Folgenden möchte ich mit einem neutraleren Begriffspaar arbeiten, und beschränke mich auf die Konzepte Bewerter vs. Ratifizierer. Dadurch gelangen wir zur folgenden Arbeitshypothese bezüglich der Rollenverteilung zwischen Bewerter und Ratifizierer je nach Sprechakttyp:

\begin{tabular}{|l|l|l|l|}
\hline \multicolumn{1}{|c|}{ Assertionen } & \multicolumn{1}{c|}{ Fragen } & \multicolumn{1}{c|}{ Befehle } \\
\hline Sprecher & $\begin{array}{l}\text { Bewerter (epistemischer } \\
\text { Richter) }\end{array}$ & Ratifizierer & $\begin{array}{l}\text { Bewerter } \\
\text { (deontischer Richter?) }\end{array}$ \\
\hline Hörer & Ratifizierer & $\begin{array}{l}\text { Bewerter } \\
\text { (epistemischer Richter) }\end{array}$ & Ratifizierer \\
\hline
\end{tabular}

\subsection{Illokutive Varianz und semantische Konstanz: MP doch als Testbeispiel}

Doch ist in den drei genannten Sprechakttypen zulässig, und es herrscht großer Konsens über seinen illokutiven Wert. In Assertionen steht doch für einen Widerspruch im Wissenshintergrund (GAST 2008). Der Sprecher/Bewerter nimmt die Tatsache zur Kenntnis, dass er den

Zitiert werden auch Beispiele aus Gelegenheitslektüren oder aus anderen wissenschaftlichen Texten, vor allem dem korpusbasierten Partikelwörterbuch von MÉTrich et al. (1995-2002).

3 Zur Verordnung der Satzmodi nach Epistemik und Deontik s. Zaefferer (2001), Truckenbrodt (2006) und Gutzmann (2011). 
Gehalt $\mathbf{p}$ als Teil des (C)ommon (G)rounds betrachtet hatte, dass dies aber beim Hörer/ Ratifizierer nicht der Fall ist. Zumindest tendenziell ist gerade der Gehalt nicht-p in den Augen des Hörers/Ratifizierers Teil des CG (s. Bsp. 17 oder 20 unten).

Doch kommt in Fragesätzen nur selten vor. Nach Schoonjans (2013) ist der interrogative Gebrauch von doch typisch für Erinnerungsfragen, bei den der Sprecher die Antwort vorher gekannt und inzwischen vergessen hat.

(3) Wie war doch gleich Ihr Name? (zit. Schoonjans 2013: online)

Andere Beispiele entsprechen Fällen, in denen der Sprecher mit dem bloßen Akt des Fragens einen Vorwurf an sich ziehen könnte:

(4) Ach HErr, HErr, warum hast du doch dieses Volk über den Jordan geführt? (zit. Schoonjans 2013: online)

Beim interrogativen Gebrauch ist es der Sprecher, der die Schuld für eine tadelhafte Unkenntnis auf sich nimmt, weil die Antwort $\mathbf{p}$ in den Augen des Adressaten als Teil des CG gelten dürfte. Diese Umkehrung ist vergleichbar mit dem Phänomen, das bei wobl zu bemerken war. Eine Modellierung auf der Grundlage von modalen Instanzen kann also auch für intersubjektive MPn eine sprechaktsortenspezifische Varianz in Kauf nehmen, die eine Beschreibung mit Sprechaktteilnehmern unerklärt ließe. Was nun mit Imperativ- bzw. Befehlssätzen? ${ }^{4}$

Illokutionsmäßig sind mehrere Untertypen unterscheidbar: Ein doch-Imperativsatz kann sowohl ein Vorwurf sein (Bsp. 5), als auch eine Ermutigung (Bsp. 6, 7):

(5) Geh redens doch nicht immer so geschwollen daher (zit. MÉTRICH et al. 2002, II: 135)

(6) An der Tür blieb er stehen. „Pardon“, sagte er, „es muss ein Irrtum sein. Man hat mich hier ins Haus gewiesen, aber ich komme nämlich geschäftlich““ Diederich hatte sich besonnen. „Ich kann es mir denken, aber das macht nichts, bitte, treten Sie doch näher. Doktor Heßling ist mein Name. Hier ist meine Mutter und meine Schwestern Emmi und Magda." (Heinrich Mann, Der Untertan, 2011: 171-172)

(7) Waren Sie es leid, dass Kinder aus Hartz-IV-Familien in aller Regel als Verlierer dargestellt werden, oder hat die Redaktion gesagt, Mensch, Undine, schreib doch mal deinen Werdegang auf, das ist doch spannend ? (DR 230813-02)

Sowohl in Fragen als auch in Befehlen sind für eine und dieselbe Kombination aus Satzmodus und MP jeweils mehrere Illokutionstypen möglich. Das gilt auch für andere MPn in anderen Satzmodi und legt nahe, dass die Distribution von MPn nicht streng illokutionssortenspezifisch ist, auch wenn das Vorkommen einer MP die illokutive Interpretationsbreite des Satzmodus reduziert. Dieser Sachverhalt entspricht den Ansichten kompositioneller Satzmodus-Theorien à la Truckenbrodt (2006) oder Gutzmann (2015). Alle formalen

\footnotetext{
${ }^{4}$ Ich verwende den Terminus Befehlssatz als Oberbegriff für Sätze, die einen streng deontischen Sprechakt ausdrücken, auch wenn wir sehen werden, dass es sich dabei nicht immer um Befehle im engeren Sinne handelt. Den Terminus Imperativsatz benutze ich nur für diejenigen Befehlssätze, die eine imperativische Verbform aufweisen.
} 
Charakteristika eines jeweiligen Satzmodus haben demnach jeweils eine eigene Bedeutung, die auf einen präzisen Faktor der Interaktion hinweist. Solche Ansätze lassen sich mit den Befunden der nicht-Searleschen Interpretationsfamilie von Austins Unterscheidung zwischen Lokutionärem und Illokutionärem vereinbaren:

„The 'locutionary act' is the act of making an utterance which, in virtue of its meaning, presents a certain illocutionary act as being performed (whether or not the act in question is actually performed).

The 'illocutionary act' is the illocutionary act actually performed (whether or not it corresponds to what the sentence encodes)" (RECANATi 2013: 4).

Der Satzmodus, als obligatorischer Teil einer Äußerung, ist im lokutiven Akt schon einbezogen. Die Bedeutung des Satzmodus ist der indizierte bzw. ideelle illokutive Akt, dessen Durchführung durch das Hervorbringen des lokutiven Aktes unternommen wird. Ironie und indirekte Sprechakte sind Fälle, in denen der wirkliche illokutive Akt dem indizierten illokutiven Akt nicht entspricht. Recanati (2013) unterstreicht, dass die formalen Marker der ,illokutiven Kraft" (= der Satzmodus) eigentlich nur für die lokutiv indizierte illokutive Kraft stehen, die unterspezifiziert bleibt. Daher die Vielfalt der illokutiven Subtypen für ein und denselben Satzmodus.

MPn sind als lokutive Satzmodusspezifizierer aufzufassen. Sie schränken den „lokutiven Kontext" und damit die Breite der illokutiven Interpretationen ein. Die sprechaktbedingte Varianz in der Interpretation von MPn ist somit auf zwei Ebenen verortet: (1) Je nach Satzmodus werden die modalen Rollen den verschiedenen Sprechaktteilnehmern zugeordnet; (2) Die illokutive Interpretation wird von der Zusammenfügung spezifizierender lokutiver Marker bestimmt, auch wenn es wohl kaum je zu einer vollständigen Festlegung auf eine einzige Illokutionsmöglichkeit kommt. In den folgenden Abschnitten soll es nun darum gehen, für jede MP mit zulässigem Gebrauch in streng deontischen Kontexten diese doppelbödige Varianz zu beschreiben.

\section{Semantisch-pragmatischer Beitrag der Modalpartikeln zu deontischen Illokutionsformen}

\subsection{Betontes $J A$}

$J A$-Befehlsätze können entweder eine V1- oder eine Vletzt-Struktur aufweisen:

(8) Dass du dir nur ja nicht das Genick verrenkst! (zit. MÉTRICH et al. 1995-2002: IV.133)

(9) Also, ich will nicht ohne Satire leben. Hört ja nicht auf damit! Versprochen? (TAZ, 07.09.1995, 14, zit. Kwon 2005: 39)

(10) So warnt Moderator Kloeppel seine Zuschauer: Nehmt die Parolen nur ja nicht ernst! (TAZ, 03.04.1996, 15, loc. cit.)

(11) Kaum ist seine Gruppe angespült, hebt er drohend den Finger: Daß Sie mir nachher ja nicht die Fähre verpassen! Sie müssen sonst hier übernachten." (TAZ, 26.08.1995, 11, loc. cit.) 
Für Truckenbrodt und Gutzmann verlangen VLetzt-Sätze nicht unbedingt eine Reaktion des Sprechers. ${ }^{5}$ Die Verbbewegung steht für die dialogale Einbeziehung des Hörers. Intersubjektive MPn, als Marker einer Antizipation über die Ratifizierung des Inhaltes der Äußerung, dürften mit dem Signifikat der Verbbewegung interagieren, vor allem wenn unter „dialogale Einbeziehung des Hörers“ die getrennte Instanzierung der beiden Rollen Bewerter und Ratifizierer verstanden wird. ${ }^{6}$

VLetzt-Befehle mit $J A$ sind angesichts der Korpusbeispiele von MÉTRICH et al. (2002) und Kwon (2005) vorzugsweise negative Befehle, also Ermahnungen, eine Handlung zu unterlassen. Es geht nicht darum, etwas zu tun, sondern die Sachen so zu lassen, wie schon sind, wobei der Sprecher den Befehl selbst anstelle des Hörers ratifiziert. Daher auch die Angemessenheit von JA-Sätzen für Befehle, die keine Agenskontrolle voraussetzen:

(15) Dass du dir nur ja nicht das Genick verrenkst! (zit. MÉTRICH et al. 1995-2002: IV.133)

Diese Kombination entspricht einer Konversationsszene, in welcher der Sprecher auf eine sofortige Akzeptanz pocht, weil der Befehl in einem Kontext erteilt wird, wo kein Raum für Gegenstandpunkte besteht. Dass es sich vorwiegend um negative Befehle handelt, erklärt auch die Rolle des kontrastiven Akzents ( $J A$ anstelle von ja) als Marker der Abgrenzung zwischen beiden Alternativen (s. Meibauer 1994: 132-170, Modicom 2016: 462-470).

\section{2. eben und halt}

Mit eben/halt geht es nicht darum, den Befehl als unwiderstehlich darzustellen. Der Hörer tendiert schon zur Durchführung der Handlung, und der „Befehl“ ist eine Bekräftigung dieser Tendenz durch den Sprecher.

(16) Den ganzen Tag zittere ich schon vor dem Heimweg. Ich hab solche Angst. Wenn ich drauftrete, könnt ich schreien vor Angst!

- Dann pass eben auf! (II.182)

(17) Und womit sollen wir spielen ? habe ich gefragt. Wir haben doch keine Zettel!

- Na ja, hat Georg gesagt, wir haben doch alle ein paar Groschen in der Tasche. Spielen wir eben mit Geld - es ist ja nicht ganz dasselbe, aber wir tun einfach so, als wenn es Zettel wären, klar? (II.182)

(18) Sie hatten mir meine Platten böse zerkratzt und auch den Plattenspieler schließlich kaputtgemacht. Tanzen wir halt ohne Musik, sagten sie und tanzten noch wilder auf dem Tisch herum. (III.70)

(19) Karoline : Ich möcht jetzt mal mit der Achterbahn fahren.

Schürzinger : Das ist ein teurer Spaß.

Karoline : Aber jetzt bin ich auf dem Oktoberfest und ich hab es mir vorgenommen. Geh fahrens halt mit! (III.70)

5 Dies ist vor allem für VLetzt-Fragen klar, aber Gutzmann betont auch, dass VLetzt-Befehle nicht wirklich adressiert sind. Erwartet wird eine stillschweigende Durchführung des Befehls ohne Diskussion.

6 In Modicom (2016:478-492, 506-510) habe ich argumentiert, dass diese getrennte Instanzierung vor allem für Exklamativsätze nicht unbedingt vorliegt. 
Dieser Wert entspricht dem MP-Gebrauch von eben in Assertionen. Dort signalisiert die MP, dass der Sprecher den Gehalt der Äußerung für tendenziell akzeptabel oder präkonstruiert hält: Eben/Halt ist demnach ein „schwaches Ja“. In Bsp. (20) signalisiert eben, dass die Neigung der Sprecherin fürs Singen der Hörerin nicht unbekannt ist. Das wird im folgenden Satz bekräftigt, wobei doch das Bestehen einer Inkongruenz bezüglich der Annahme dieser Prämisse markiert. Schließlich erfolgt der halt-Befehlssatz: Die Sprecherin hat um diesen Zeitpunkt betont, dass das Nicht-Gönnen in ihren Augen die Prämissen ihrer Beziehung mit der Hörerin verletzt. Sie erwartet eine umstandslose Ratifizierung ihre Bitte:

(20) Anna, das darfst du nicht. Ich sing eben gern. Das weißt du doch. Gönn’s mir halt, Anna. (III.70)

\subsection{Rubig}

Das Signifikat von Adj. ruhig beinhaltet den Begriff der Vollendung eines Prozesses ohne Störfaktor, der den Durchlauf der Handlung oder die Tätigkeit eines Handlers bedrohen könnte. Das einzige Vorkommen von MP ruhig in meinem Korpus erfolgt nicht in einem Befehlsatz, sondern in einem Deklarativsatz mit deontischem Modalverb - womöglich der Übergangskontext zwischen Adv. rubig und MP rubig.?

(21) Und dann sagt der Vermieter, oh ja, jetzt haben wir gerade eine Wohnungssituation, die sehr, sehr knapp ist, da kann ich ruhig 70 Euro mehr nehmen für die Wohnung als Beispiel. (DR 050913-06)

Ein Befehlssatz unter Angabe von ruhig ist oft eine Ermunterung oder eine Erlaubnis: Der Sprecher beseitigt jedes Hindernis aus dem Wege des Hörers, der dadurch die Handlung durchführen kann und soll (s. MÉTRICH et al. 2002, IV.28). Im Stile der „Theorie der möglichen Welten“ entspräche dies einer Spezialisierung für deontische Möglichkeit (anstelle der deontischen Notwendigkeit als Defaultwert der Befehlssätze). Hinter dem Bewertungsakt steht (illokutiv gesehen) die öffentliche Ratifizierung einer dem Hörer zugeschriebenen Absicht, wie an Bsp. (22) zu sehen ist.

(22) Mönch : Ich verstehe Ihre Bitterkeit. Sie denken an die gewissen außerordentlichen Machtmittel der Kirche.

Galilei : Sagen Sie ruhig Folterinstrumente. (Brecht, Leben des Galilei, zit. MÉTrich et al. 2002, IV.32)

Galilei signalisiert, dass er das Gemeinte verstanden hat, dass die Bezeichnung „Folterinstrumente“ auch in seinen Augen angemessener wäre, und dass jede gesellschaftliche Norm, die den Gebrauch des Wortes hindert, in diesem Kontext aufgehoben gehört. Der Austausch erfolgt in mehreren Zügen:

7 Es mag den Leser wundern, dass dieser Deklarativsatz in einem Aufsatz über „Befehlssätze“ behandelt wird. Illokutionsmäßig gehe ich davon aus, dass dieser Satz einem (permissiven) deontischen Sprechakt entspricht. Gerade an solchen Beispielen wird das - für diese Untersuchung so wichtige - Spannungsverhältnis zwischen Illokution und Satzmodus besonders anschaulich. 
Zug 1: Der Mönch will von Folterinstrumenten reden, was in seinen eigenen Augen aus Gründen der sozialen Akzeptabilität nicht nachdrücklich erfolgen kann.

Zug 2: Er benutzt eine euphemistische Ausdrucksweise.

Galilei kann entweder auf Zug 1 oder auf Zug 2 reagieren. Er macht beides:

Zug 3: Galilei gibt zu wissen, dass er Zug 1 hinter Zug 2 erkannt hat, und dass das Hindernis, das auf dem Wege des Mönches stand, für ihn keine Geltung hat.

Denken wir an die Unterscheidung zwischen Lokution und Illokution zurück, so können wir sagen, dass Galilei die illokutive Absicht des Mönchs gerade dadurch ratifiziert, dass er seinen lokutiven Akt zurückweist.

\section{Modale Grenzfälle? Nur und mal in Befehlssätzen}

\subsection{Nur}

In ihrem Eintrag zu MP nur schreiben MÉTRICH et al. (2002: III.35), dass der Sprecher beim Gebrauch von nur "großen Wert darauf legt, dass seiner Ermahnung Folge geleistet wird“ (,le locuteur attache une grande importance au fait que son exhortation soit suivie d'effet"). Neben diesem Hinweis, dass an eine illokutive Verstärkung des Befehls denken lässt, merken die Autoren des korpusbasierten Lexikons auch an, dass die deontischen nur-Sätze meistens negativen Befehlen entsprechen (loc. cit.).

(23) Vergiss nur nicht bei dem Wäschegeschäft vorbeizugehen, die Sachen müssen längst fertig sein. (zit. MÉTRICH et al. 2002: III.352)

(24) Glaube nur nicht, dass ich mich in euren Weg stellen will. (ibid.)

Dass hier kein formaler Zwang vorliegt, sondern höchstens eine illokutive Affinität, sieht man am affirmativen Imperativ Lass nur!, der ebenfalls eine Ermahnung zum Nicht-Handeln ist. In dieser Hinsicht lässt sich eine Parallele zwischen nur und ja ziehen.

(25) A : Soll ich dir welchen [Kaffee $]$ machen?

B : Ach, lass nur, sagte ich. Ich trinke etwas Sprudel, so wichtig ist das nicht. (op.cit., 353)

Fest steht auch, dass die Fokuspartikel nur, wenn sie die hinreichende Bedingung ausdrückt und nicht-exklusiv interpretiert wird, keiner eigenen Fokuskonstituente bedarf (s. KöNIG 1991 und Modicom / Duplâtre 2018). Es ist also fraglich, ob wir es wirklich mit einer MP zu tun haben, oder mit einem fokusfreien Gebrauch der Fokuspartikel. Dieser Ausdruck der hinreichenden Bedingung durch nur geht oft mit einer minimisierenden Wirkung einher (die wiederum ironisch gedeutet werden kann, aber nicht muss). Das sieht man zum Beispiel am Satzverknüpfer nur:

(26) Peter möchte Tänzer werden, nur hat er ein Holzbein.

Die minimisierende Wirkung ist in mehreren Fällen von nur-Befehlen annehmbar: 
(27) Auch ein Sturz des Regimes würde Syrien in, in, in einen vertieften Bürgerkrieg stürzen, weil die Opposition so zerstritten ist, weil Reste des Regimes weiterkämpfen werden. Stellen Sie sich nur vor, dass die Chemiewaffenbestände dann außer Kontrolle geräten, dass verschiedene ä Milizen oder Teile des ehemaligen Regimes dieses Waffen kontrollieren. (DR 020913-03)

Argumentativ ist (27) ein Fall von argumentum a fortiori, wo eine minimale Bedingung ausreicht, um den ersuchten Effekt zu bewirken, wobei die Tür offengelassen wird für stärkere Bedingungen, die noch dramatischere Folgen hätten. Auf semantischer Ebene lässt sich dieses nur als ein Zeichen der hinreichenden Bedingung identifizieren.

In negativen Befehlen nimmt der Sprecher in Kauf, dass der Hörer und er divergierende deontische Ansichten haben. Durch den Gebrauch von nur wird der Gegensatz manchmal abgeschwächt, manchmal aber verstärkt. Die minimisierende Wirkung entspricht der Interpretation der Partikel als Ausdruck der hinreichenden Bedingung. Für die Fälle, in denen der Befehl durch das Vorkommen von nur eindringlicher wirkt, tritt die exklusive Lesart ("notwendige Bedingung") in den Vordergrund, zumal diese Dramatisierung oft mit einer Betonung der Partikel einhergeht (AвRA ham 2017: 189-190). Da betonte Partikeln einen Grenzfall von Kontrastfokus darstellen (Meibauer 1994, Modicom 2016: 462-470), ist die Hypothese einer remanenten Exklusivität bei betontem nur naheliegend. Dabei haben wir den Rückgriff auf modale Instanzen hier nicht nötig: Die Varianz in der Interpretation der Partikel lässt sich mit einem Umweg über die Fokuspartikel erklären. Der MP-Status von nur ist nicht außer Frage (ABRAн Am 2017: loc. cit.), und die Beschreibung der Varianzparameter scheint das zu bekräftigen: Nur ist weniger eine MP, als eine Fokuspartikel mit verschiedenen Fokus- und Skopus-Möglichkeiten.

\subsection{Mal}

Genauso zweifelhaft ist der MP-Status von mal. Wiederum liefern MÉTRICH et al. (2002, II.237) einen interessanten Hinweis: Mal könne mit „Handlungen“ (frz. Actions) benutzt werden, aber nicht mit „Einstellungen“ (frz. Attitudes).

$(28)^{*}$ Verzeih' ihm mal! ${ }^{8}$

(29) Ja zeig mal, was du in deiner Tasche hast!

Wenn es eine Präferenz für (telische? agenskontrollierte?) Handlungsverben gibt, haben wir es eigentlich mit einer Aktionsartpartikel zu tun. Auch hier scheint der Rückgriff auf modale Instanz verzichtbar zu sein, wenn es darauf ankommt, die illokutive Varianz von Mal-Befehlen zu deuten. Der Ursprung von mal ist das Zeitadverb einmal, und sein Signifikat beinhaltet schon die beiden Bedeutungskomponenten, deren Zusammenspiel die kontextuelle Varianz von mal umfasst: Einmal kann entweder im Sinne von Ein für allemal gedeutet werden, oder im Sinne von Nur ein mal.

8 Dabei kann das Verb verzeihen auch eine „Handlung“ bezeichnen (z.B. den Sprechakt der Verzeihung). Es ist dann zu erwarten, dass Verzeih ihm mal akzeptabel wird. 
Einmal im Sinne von Ein für allemal bezeichnet ein präzises, identifizierbares Mal, d.h. ein Vorkommnis des Vorgangs mit Vollendung des Vorgangs (Kulmination). Der intersubjektive Wert dieser terminativen Lesart ist, dass dem Hörer unterstellt wird, er tendiere nur zur Vollendung des Vorgangs oder sei dabei eingehemmt. Die Nur ein Mal-Lesarten (wo nur Ausdruck der hinreichenden Bedingung ist) bezeichnen die Durchführung der Handlung als eine geringe Herausforderung, die man schnell hinter sich bringen kann. Die beiden Werte sind kontextgebunden, und nicht unbedingt exklusiv voneinander, wie am folgenden Beispiel zu sehen ist:

(30) Also, ich so eine Permafrostzone macht man ja bei bestimmten Baumaßnahmen. Aber die hilft natürlich nur, wenn sie dauernd aufrecht erhalten wird. Fragen Sie mal, ob das 20 Jahre wirklich geht. (DR 030913-02)

Die Grundlage hier ist wohl die nur ein Mal-Lesart (vergleichbar zu Fragen Sie nur...): Eine einfache, minimale Frage reicht, um des Pudels Kern zu identifizieren. Aber diesen Schritt muss man erst vollziehen, und hier schwingt die erste Komponente mit.

\section{Fazit}

Nach diesem Überblick können wir festhalten, dass eine strenge semantische Kennzeichnung durch das modale Paar Bewerter / Ratifizierer in Befehlsätzen nur für doch, ja, eben/halt und rubig haltbar ist. Für nur und mal ist dieser Beschreibungsansatz weniger relevant, was mit der Remanenz einer anderen, nicht-modalen Partikellesart zu tun haben dürfte.

Dabei bleibt, dass der MP-Gebrauch in Befehlssätzen einer illokutiven Spezialisierung entspricht, mit verschiedenen Abschattungen deontischer Modalität. Der Beitrag von MPn in solchen Äußerungen kann an denselben Standards gemessen werden, wie ihre Leistung in Deklarativ- und Fragesätzen (d.h. in epistemischen Satzmodi). MPn sind nicht zwangsläufig epistemisch. Die Art der Modalität, die von intersubjektiven MPn spezifiziert wird, ist abhängig von anderen modalen Operatoren im Satz, allen voran vom Satzmodus.

\section{Bibliographie}

Abraham, Werner (2017): Modal particles and Verum focus: New corollaries. In: Fedriani, Chiara / SAnso, Andrea (Hg.): Pragmatic Markers, Discourse Markers and Modal Particles: New perspectives. Amsterdam: John Benjamins, 171-202.

Doherty, Monika (1985): Epistemische Bedeutung. Berlin: Akademie-Verlag.

GAST, Volker (2008): Modal particles and context updating: The functions of German 'ja', 'doch', 'wohl' and 'etwa'. In: VATER, Heinz / Letnes, Ole (Hg.): Modalverben und Grammatikalisierung. Trier: Wissenschaftlicher Verlag, 153-177.

Gutzmann, Daniel (2011): Ob einer wohl recht hat? Zwei Satzmodustheorien für das Deutsche im Vergleich. In: Deutsche Sprache 39-1, 65-84. 
Gutzmann, Daniel (2015): Use-conditional meaning. Studies in multidimensional semantics. Oxford: Oxford University Press.

KöNIG, Ekkehard (1991): The meaning of focus particles. A comparative perspective. London: Routledge. Kwon, Min-Jae (2005): Modalpartikeln und Satzmodus: Untersuchungen zur Syntax, Semantik und Pragmatik der deutschen Modalpartikeln. Diss. der LMU München.

Meibauer, Jörg (1994): Modaler Kontrast und konzeptuelle Verschiebung. Studien zur Syntax und Semantik deutscher Modalpartikeln. Tübingen: Niemeyer.

MÉTRICH, René / FAUCHER, Eugène / Courdier, Gilbert (1995-2002): Les Invariables difficiles. Dictionnaire allemand-français des particules, connecteurs, interjections et autres mots de la communication. Nancy: Nouveaux Cahiers d'Allemand. 4 Bde.

Modicom, Pierre-Yves (2016): L'énoncé et son double: Recherches sur le marquage de l'altérité énonciative en allemand. Paris: Diss. der Universität Paris-Sorbonne.

Modicom, Pierre-Yves / Duplâtre, Olivier (2018): Nicht nur Fokuspartikel: Geschichte und Varianz von nur im Deutschen. In: Gautier, Laurent / Modicom, Pierre-Yves / Vinckel-Roisin, Hélène (Hg.): Diskursive Verfestigungen. Berlin: De Gruyter, 73-92.

Recanati, François (2013): Content, Mood, and Force. In: Philosophy Compass 8, 622-632 (zitiert wird aus der Fassung unter URL: https://jeannicod.ccsd.cnrs.fr/file/index/docid/782906/filename/Force_and_content9.pdf [21.12.18])

Schoonjans, Steven (2013): Zur französischen Übersetzung der deutschen Modalpartikel doch: eine satztypübergreifende Analyse. In: Studii de gramatica contrastiva 19, 85-110.

TruCKenbrodt, Hubert (2006): On the semantic motivation of syntactic verb movement to C in German. In: Theoretical Linguistics 32, 257-306.

ZAefFerer, Dietmar (2001): Deconstructing a classical classification: a typological look at Searle's concept of illocution type. In: Revue Internationale de Philosophie 216, 209-225.

Zimmermann, Malte (2008): Discourse particles in the left periphery. In: SHaer, Benjamin / Cook, Philippa / Maienborn, Claudia / Frey, Werner (Hg.): Dislocated elements in discourse. Syntactic, Semantic, and Pragmatic Perspectives. London: Routledge, 200-231. 